\title{
MODELO HOLÍSTICO DE METAESCRITURA
}

\author{
Yamid Fabián Hernández Julio* \\ Yasmin Osmany Serpas Marimón** \\ Socorro Nohemy Carrascal Torres***
}

RESUMEN: Este artículo propone un programa holístico de metaescritura, el cual está conformado por cinco componentes: cognitivo, metacognitivo, afectivo-emocional, social y competencia textual, el substrato teórico se halla en los aportes de Van Dijk, Díaz, Flower y Hayes, entre otros. El método fue acción-participación. Se realizó en tres fases. 1. Diagnóstico: se identificaron los niveles de desempeño de la competencia textual de los estudiantes; 2. Diseño: Se usaron los ciclos característicos del método para el diseño y 3. Se identificaron las transformaciones producidas a raíz de su aplicación. Los resultados evidencian que la metodología implementada, ayudó a los estudiantes a emancipar pensamientos y sentimientos reprimidos, crear nuevas ideas y/o mundos, sentir que estaban vivos y que alguien los escuchaba a través de la voz de las letras. Igualmente, obtuvieron un aumento en su nivel de desempeño en la competencia textual, todo esto a través del uso de la Metacognición.

Palabras-clave: Metacognición. Modelo Holístico. Metaescritura.

\footnotetext{
* Magíster en Educación SUE Caribe Universidad de Córdoba (Colombia) y Profesor Catedrático Universidad de CórdobaColombia Categoría Auxiliar. E-mail: yafaheju@hotmail.com

** Magíster en Educación. SUE Caribe Universidad de Córdoba (Colombia) y Profesora de Lengua Castellana e Inglés del Centro Educativo "Mimbres" de Ciénaga de Oro. E-mail: jasminserpa@yahoo.com

*** Dra. En Psicología y educación. Universidad de Granada (España) y Profesora Tiempo Completo Facultad de Educación y Ciencias Humanas de la Universidad de Córdoba (Colombia). E-mail: ncarrascal@gmail.com
} 


\section{AN HOLISTIC MODEL OF META-WRITING}

ABSTRACT: This article proposes a holistic program of meta-writing, its methodology being comprised of five components: cognitive, meta-cognitive, affective, emotional, social; it also examines textual competence, its theoretical background lying in the contributions of Van Dijk, Diaz, Flower \& Hayes, etc. The method was one of action-participation. It was realized through three phases. 1. Diagnosis: The level of performance, in relation to each student's textual competence, was evaluated, 2. Design: The cycles, characteristic of this method, were used to develop the program and 3 . The changes produced at the most fundamental level, were identified. The results showed that the methodology implemented, helped students to liberate repressed thoughts and feelings, to create new ideas and/or worlds, to feel that they were alive and that someone could hear them through the their self-expression in writing. Likewise, they had an increased text competence level when performing the writing exercises, resulting from the use of Meta-cognition.

Keywords: Meta-cognition. Holistic Model. Meta-writing.

\section{El problema}

La escritura es un proceso complejo, cuya enseñanza y aprendizaje se ha constituido en una de las grandes dificultades en nuestras escuelas, éste proceso no se ha realizado de la manera efectiva, porque se evidencia en los bajos resultados obtenidos en las pruebas evaluativas nacionales colombianas (Pruebas SABER (2005-2006) e ICFES (2007-2008)), e Internacionales (Programa Internacional de Evaluación de Estándares [PISA] (2006)). Según Pérez (2003), a partir de los resultados presentados en la prueba SABER - nacionales - se identificaron cinco grandes dificultades en el área de escritura:

No hay producción de textos, hay escritura oracional.

No se reconocen diferentes tipos de texto.

Falta cohesión en los escritos de los estudiantes.

No se usan los signos de puntuación en los escritos.

No se reconocen las intenciones de la comunicación.

Se han identificado varias razones por las cuales se pueden presentar las dificultades en escritura; la primera se encontró en Lomas (1999, p. 324, citado en LACON y ORTEGA, 2004, p. 1) quien señala que una de las principales dificultades al abordar el trabajo con textos escritos, en 
la educación media, es la "ausencia de una metodología específica para la enseñanza de la lengua escrita". La primera característica es que el enfoque que guía su enseñanza, es el oracional (oración como principal unidad de estudio), y alrededor de la cual se organizan muchos de los contenidos que se imparten en la asignatura de lengua castellana.

La segunda característica de la enseñanza de la escritura, es que se ha llevado a cabo aislada del contexto, de la realidad social en la cual se encuentra el estudiante, ésta situación se describe en el siguiente fragmento: "el manipular unidades sintácticas sin un sentido de contexto que las conecte a modo de una relación significativa puede a la larga ser contraproducente" (ROSENBLAT, 1996 p. 54). Y es precisamente las exigencias del contexto, lo que determina la necesidad de trabajar la escritura en la escuela. "La escritura es importante en la escuela porque es importante fuera de ella" (FERREIRO, 1982).

La tercera característica se refiere a la desconexión de los procesos de pensamiento, y al desconocimiento que es un proceso cognitivo (FLOWER; HAYES, 1996; MORALES; FONSECA; MORALES; MORALES, 2006, p. 37; CASTILLO; TRIANA; DUARTE; PÉREZ; LEMUS, 2007; RODRÍGUEZ, 2009, p. 1) por lo cual se ha enfatizado en la reproducción y no en la creación de escritura. Al respecto, Emilia Ferreiro (2002) señala que lo que se trabaja como escritura se refiere sólo a ejercicios mecánicos de copiado, es decir, que los estudiantes no están construyendo sus propios textos o "textos libres" donde se vean desafiados a usar sus procesos de pensamiento y conocimientos previos.

Y la última característica de la enseñanza de la escritura es que no se incluye la parte afectiva del sujeto que escribe, no se tiene un lugar, un espacio en el universo de la escritura; ahora, si no tenemos un espacio en ella, ella tampoco lo tiene en nosotros, esto se evidencia en el texto a continuación:

En A place called school [Un lugar llamado Escuela], Goodlad escribe: "Cosas que para los alumnos son preocupaciones primarias de la vida cotidiana son percibidas por los maestros como una disonancia dentro de lo que es propio del aula y de la escuela" (GOODLAD, 1984, citado en MCCORMICK, 1998).

Esto indica que la vida de los estudiantes está excluida de los procesos escriturales que se imparten en la escuela, esto se presenta en la realidad colombiana, a pesar, que diversos autores colombianos han sus- 
tentado lo esencial que resulta incluir su vida al trabajar la escritura, entre éstos Peña, (2003, p. 5) quien afirma: "Si hay un axioma fundamental de la escritura es que es algo que sale de dentro del escritor: de un impulso interno, de una emoción, de un interés, de una pregunta, de una experiencia de vida o de conocimiento, o de algo que se ha imaginado".

Para Díaz (1999), si la palabra es un ente, fonético, gráfico, semántico, morfológico y pragmático, ¿Por qué nuestra enseñanza de la escritura solo abarca los dos primeros aspectos?, - que se imparten en los primeros años de escolaridad colombiana- donde el niño logra establecer la relación grafía-sonido. Más tarde en primaria se le enseña reglas de ortografía, las partes de la oración, y los contenidos morfológicos. En este tipo de enseñanza prevalece: "la explicación de reglas gramaticales en el vacío; análisis morfosintáctico de oraciones aisladas del contexto; adiestramiento en la correcta utilización de signos de puntuación, mayúsculas, normas ortográficas y análisis de errores que se cometen al escribir" (DÍAZ, 1999, p. 73).

$\mathrm{Al}$ iniciar el análisis de las razones por las cuales se presentan las dificultades en escritura, no se incluyó un componente que luego, a través de las lecturas surge como imprescindible si se quieren mejorar los procesos escriturales, éste componente es la metacognición. Se plantea entonces, la necesidad de establecerla como un eje alrededor del cual construir propuestas para la enseñanza de la escritura.

Teniendo en cuenta las problemáticas anteriores, surge la intención de proponer una nueva práctica en el aula de clases, para ello es necesario interrogarnos: ¿Qué cambios promueve el desarrollo de un programa holístico de metaescritura orientado a mejorar la competencia textual y emocional de los estudiantes de noveno grado del centro educativo "Mimbres" de Ciénaga de Oro?

\section{Objetivos}

Estos fueron los objetivos de la investigación:

\section{Objetivo general}

- Diseñar y desarrollar un programa holístico de metaescritura orientado a mejorar la competencia textual y emocional en los estudiantes de noveno grado del centro educativo "Mimbres" de Ciénaga de Oro. 


\section{Objetivos específicos}

- Identificar en la población sujeto de estudio los niveles de desempeño efectivo en la competencia textual, como insumo para la formulación de estrategias de mejoramiento.

- Desarrollar el plan de acción del programa holístico de metaescritura orientado a mejorar la competencia textual y emocional en los estudiantes.

- Identificar en la población sujeto de estudio las transformaciones en la competencia textual y emocional a partir del desarrollo del programa holístico de metaescritura.

\section{Lo que se realizó}

Como respuesta a lo anterior se realizaron propuestas de dos tipos: teóricas y metodológicas. Referente al aspecto teórico, se propuso una definición y un modelo holístico de metaescritura. Esta necesidad de orientar estudios hacia la construcción de teorías integradoras, que abarquen las diferentes dimensiones, es propuesta por Witte (1992, citado en MARINKOVICH, 2002), quien "plantea que una teoría de la escritura necesita sintetizar los factores cognitivos, sociales y lingüísticos o textuales". (p. 5).

El equipo investigador propone un modelo que además de las dimensiones anteriores, le incluye dos más, la afectiva y la metacognitiva, el cual busca responder a las deficiencias planteadas en la descripción del problema. (Gráfico 1)

GRÁFICO 1. Componentes del modelo holístico de Metaescritura

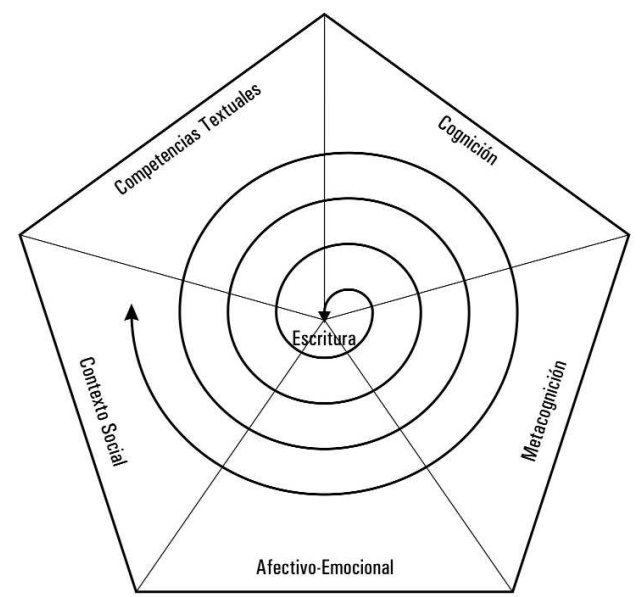


La Dimensión Cognitiva: Respecto a este punto, se explican tres aspectos en que la escritura está relacionada con lo cognitivo: primero, que ella es un proceso cognitivo, lo cual esta soportado por numerosos teóricos (GRAVES, 1984; KROLL, 1981; FLOWER; HAYES, 1983, citados en MARINKOVICH, 2002), pero se ha elegido la teoría de la redacción como proceso cognitivo, propuesta por Linda Flower y John Hayes (1996). La cual contempla tres elementos que influyen en la escritura, el problema retórico, el texto escrito y la memoria a largo plazo del escritor, y tres grandes procesos que constituyen el acto de escribir, éstos son: la planificación, la traducción y el examen y finalmente el componente de control.

El segundo aspecto abarca por un lado la movilización de los diversos procesos de pensamiento que se ponen en juego al momento de escribir. Y por el otro, el uso que se hace de los conocimientos disponibles en su memoria. Este sería el aspecto cognoscitivo de la escritura y finalmente, un tercer aspecto que propone el psicólogo Jerome Brunner (2004), denominado narrativa del pensamiento que expresa que hay dos tipos de pensamiento, el lógico- formal o paradigmático y otro llamado el pensamiento narrativo, constituyéndose de esta manera una conjunción entre escritura y pensamiento, que abarca la expresión, construcción y reconstrucción de nuestra vida a través de la utilización de la narrativa.

El Componente Social: el componente social no estaba incluido dentro de los procesos escriturales, pero es innegable su función determinante en éstos, lo cual es sustentado por diferentes teóricos, expuestos a continuación.

Cooper, agrega "la escritura es una actividad social, dependiente de estructuras sociales" (1986, p. 336) (MARINKOVICH, 2002, p. 4-5). En esta misma perspectiva se orienta la siguiente afirmación: "El escritor siempre está realizando transacciones con un ambiente personal, social y cultural, por tanto el proceso de redacción debe ser visto siempre expresando factores tanto personales como sociales, individuales y ambientales" (ROSENBLATT, 1996, p. 39).

También Linda Flower (1993, citada en MARINKOVICH, 2002), quien inicialmente no incluía en su teoría el aspecto social, más adelante la reconoce como un factor preponderante en los procesos de escritura. Pero esta orientación social de la escritura también tiene otra conse- 
cuencia en los procesos de enseñanza en la escuela, y es la funcionalidad con que se escribe, tal como lo afirma Halliday, Martin (1989, citado en MARINKOVICH, 2002, p. 5) quien argumenta: "La escritura en situaciones escolares debe poner el acento en la conexión entre uso del lenguaje y propósito social de los textos". En otras palabras, saber usar la escritura para comprender el mundo.

Es esencial hacer énfasis en la dimensión social, ya que los estudiantes no existen en el vacío sino que están inmersos en un contexto familiar, cultural y económico que organiza y define sus vidas, del cual proceden situaciones que influyen en su vida personal y que a su vez él o ella también influye sobre éstas, presentándose entonces una relación de influencia recíproca entre individuo y contexto.

El Componente Afectivo-Emocional: El principio que guió esta investigación se denominó escritura desde y para la vida, y quiere enfatizar que el lugar desde donde se escribe debe ser la vida misma y con fines conectados a la propia vida. Esto es sostenido por diferentes autores: "La manifestación personal es el motor del proceso de escribir, la razón fundamental para escribir" (GRAVES, 1991, p. 44). "Podemos dar curso al impulso humano de escribir si ayudamos a los estudiantes a darse cuenta de que sus vidas son algo sobre lo cual vale la pena escribir, si los ayudamos a elegir sus temas, su género y su auditorio" (MCCORMICK, 1998, p. 16).

Y, finalmente, la intención debería surgir de la experiencia lingüística y vivencia real del escritor, o bien ser capaces de permitirle imaginarlas. Un propósito fundado en la experiencia personal gesta e impulsa el empuje. Las ideas vitales que nacen de situaciones, actividades, discusiones, problemas o necesidades brindan la base del proceso activamente selectivo y sintetizador de la elaboración del significado (ROSENBLATT, 1996, p. 42).

Por otra parte, todos los seres humanos nos vemos enfrentados a situaciones que nos producen angustia, dolor, tristeza, ira, y todo un cúmulo de sentimientos, que en muchas ocasiones no encuentran canales para que fluyan y sean expresados sanamente. Como una forma de trabajar esta problemática, James W. Pennebaker realizó diversas investigaciones tendientes a utilizar la escritura como una herramienta terapéutica para estimular el afrontamiento de situaciones estresantes, traumáticas y dolorosas, erigiendo la escritura como mecanismo de catarsis. 
Este enfoque terapéutico de la escritura se está utilizando con éxito con diferentes poblaciones y son justamente estos resultados los que nos animaron a implementar el trabajo en el centro educativo "Mimbres", para proporcionar a los estudiantes un recurso eficaz y disponible en cualquier momento y espacio de sus vidas, para elaborar situaciones dolorosas, estresantes y traumáticas. Este enfoque se encuentra sustentado en la definición que formula Pennebaker, (1990, citado en ESCUELA DE ESCRITORES, 2009, p. 1), "Escribir puede ser una avenida hacia un lugar interior donde podemos confrontar traumas y ponerlos a descansar, y sanar cuerpo y mente".

También en los últimos años se ha venido desarrollando un nuevo enfoque en psicología, el de Resiliencia, definida por Edith Grotberg como "la capacidad del ser humano para hacer frente a las adversidades, superarlas e incluso salir fortalecidos de ellas" (GROTBERG, 2006, p. 18), y así mismo se ha trabajado la escritura como una de las herramientas a través de la cual se puede fortalecer la Resiliencia (BRUDER, 2007). Como puede observarse, la escritura permite trabajar exitosamente varios aspectos que tienen que ver con la dimensión afectivo-emocional.

El Componente metacognitivo: La Metacognición es un concepto tomado de la psicología, fue introducido por John Flavell en la década de los 1970, para este investigador la Metacognición: "Se refiere al conocimiento que uno tiene sobre los propios procesos y productos cognitivos, o cualquier otro asunto relacionado con ellos" (BARA, 2001, p. 68).

La Metacognición es un elemento imprescindible en los procesos de escritura, cuya utilización debe estimularse desde la escuela. Un ejemplo de su utilización se muestra en el siguiente texto: "Cuando se pide a los alumnos que describan sus procesos de escritura, ...Los chicos observan sus estrategias de escritura y pronto las revisaran de modo consciente, y a su vez, ser conscientes de sus propios procesos de pensamiento les da un nuevo dominio sobre su escritura" (MCCORMICK, 1998, p. 200).

Se confirma entonces, que incluir un grado de conciencia sobre la escritura es indispensable para su desarrollo, y que la escritura se convierte en un proceso metacognitivo en la medida que logra una ejercer una conciencia y un control sobre las diferentes acciones y conocimientos implicados a la hora de escribir. 
El Componente lingüístico y competencias textuales: La concepción de la oración como unidad de estudio del lenguaje, trajo como consecuencia que los estudiantes no se les enseñara a organizar las oraciones en un texto, ni tampoco otros mecanismos que tienen que ver con el sentido de globalidad. Esto se refleja en el texto a continuación: "En la enseñanza tradicional es muy poca la atención que se la ha prestado al estudio de cómo se combinan las oraciones en el texto hasta lograr discursos escritos coherentes, cohesivos y con unidad" (DÍAZ, 1999, p. 18).

Ésto, comenzó a cambiar con la introducción de un nuevo enfoque propuesto por el lingüista Teun Van Dijk, en la década de los 1970, conocido como Textolingüística o lingüística del texto, "el cual concibe no a la oración, sino al texto como la máxima unidad de análisis lingüístico" (DÍAZ, 1999, p. 18). Éste cambio de la unidad de estudio generó la creación de nuevas competencias, las textuales, las cuales son definidas en los lineamientos curriculares de lengua española, publicado por el Ministerio de Educación Nacional (M.E.N.) de Colombia, como: "Los mecanismos que garantizan coherencia y cohesión a los enunciados (nivel micro) a los textos (nivel macro).

Además de las competencias textuales se incluyen conceptos esenciales como superestructura, macroestructura y microestructura, propuestos por Van Dijk (1983). Así mismo se tuvo en cuenta los conceptos definidos por Álvaro Díaz (1999) como las características de un texto, que son: Propósito comunicativo, Sentido completo, Unidad temática, Cohesión, y Coherencia.

La importancia del modelo holístico de metaescritura, radica en lo indispensable de tener una teoría que guíe la práctica, una claridad conceptual para poder proponer cambios en la forma como se enseña la escritura.

Respecto al aporte práctico, se propone una metodología compuesta por cuatro elementos: primero, un conjunto de talleres, con una temática y una producción textual específica; segundo, una herramienta didáctica "el formato de revisión de metaescritura"; tercero, un método para el ejercicio de la escritura, y finalmente una plantilla para evaluación de la escritura. El contenido de los diferentes talleres realizados se muestra en la tabla 1. 
TABLA 1. Talleres de producción textual

\begin{tabular}{c|c|c}
$\mathbf{N}^{\circ}$ & TEMATICA TRABAJADA & PRODUCCION TEXTUAL \\
\hline $\mathbf{1}$ & Definición de Resiliencia & Creación de un cuento \\
\hline $\mathbf{2}$ & Características y conductas resilientes & $\begin{array}{c}\text { Elaboración de artículo periodístico a partir de la película } \\
\text { "quien quiere ser millonario" }\end{array}$ \\
\hline $\mathbf{3}$ & Autoestima y autonomía & Creación de una canción \\
\hline $\mathbf{4}$ & Estrategias para resolución de problemas & Elaboración de problemas matemáticos \\
\hline $\mathbf{5}$ & Procesos de pensamiento & Resolución de talleres con diferentes procesos de pensamiento \\
\hline $\mathbf{6}$ & Metacognición & Resolución de problemas con estrategias cognitivas y metacognitivas \\
\hline $\mathbf{7}$ & Proceso de revisión metacognitiva & Reescritura del cuento a partir del formato de metaescritura \\
\hline $\mathbf{8}$ & Estrategias de afrontamiento & $\begin{array}{c}\text { Escritura de situación dolorosa, individual } \\
\text { Realización guion para obra de teatro }\end{array}$ \\
\hline $\mathbf{9}$ & Sentido de propósito y de futuro & Escritura de epístola a sí mismo, dentro de 10 años \\
\hline $\mathbf{1 0}$ & Proceso de escritura & Artículo periodístico de un asesinato ocurrido en el \\
& corregimiento los mimbres \\
\hline $\mathbf{1 1}$ & Concurso de artículos & Se realizó en forma grupal un artículo y se premiaron \\
& & varias categorías
\end{tabular}

En lo referente al formato de revisión de metaescritura. Las bases conceptuales que se tuvieron en cuenta para la construcción de este instrumento, fueron sustraídas de los planteamientos de Teun Van Dijk (1983), Álvaro Díaz (1999), Ana Atorresi (2005) y los lineamientos curriculares del M.E.N de Colombia para la Lengua Castellana (PÉREZ, 1998). El anterior sustento teórico, más la evaluación realizada por parte de expertos en el área de Lingüística (Lengua Castellana) y de Metacognición, se consideran las causas para establecer la validez del instrumento. El formato se puede observar en la tabla 2. 
TABLA 2. Formato de revisión de Metaescritura

\begin{tabular}{|c|c|c|}
\hline CRITERIOS & DEFINICIÓN & PREGUNTAS METACOGNITIVAS \\
\hline 1. Superestructura & $\begin{array}{l}\text { Es la forma global como se organizan los } \\
\text { componentes de un texto. } \\
\text { Ej. Cuento, noticia, textos argumentativos, } \\
\text { expositivos, descriptivos. }\end{array}$ & $\begin{array}{l}\text { 1. ¿A qué superestructura corresponde mi texto? } \\
\text { 2. ¿En qué modalidad lo escribí? } \\
\text { 3. ¿Tengo mi texto escrito de acuerdo a la } \\
\text { modalidad de esa superestructura? }\end{array}$ \\
\hline 2. Propósito Comunicativo & $\begin{array}{l}\text { Intención comunicativa específica por } \\
\text { la que se escribe el texto. }\end{array}$ & $\begin{array}{l}\text { 1. ¿Con qué intención escribí el texto? } \\
\text { 2. ¿Es claro para el lector, el propósito } \\
\text { comunicativo para el cual lo escribí? }\end{array}$ \\
\hline 3. Sentido Completo & $\begin{array}{l}\text { Cuando en el texto aparece suficiente } \\
\text { información para que al leerlo se pueda } \\
\text { entender el propósito por el cual se } \\
\text { escribió. }\end{array}$ & $\begin{array}{l}\text { 1. ¿Tiene el texto suficiente información sobre el } \\
\text { tema? } \\
\text { 2. ¿El lector comprende mi propósito comunicativo? }\end{array}$ \\
\hline 4. Unidad temática & \begin{tabular}{|c|} 
Cuando en el texto el tema se desarrolla \\
con una idea principal y otras secundarias, \\
además, no se incluye información que no \\
esté directamente relacionada con el tema. \\
\end{tabular} & $\begin{array}{l}\text { 1. ¿Desarrollo completamente la idea central del texto? } \\
\text { 2. ¿Tengo mi texto claramente organizado? (temas y } \\
\text { subtemas, párrafos) } \\
\text { 3. ¿Mantengo el tema a lo largo de mi texto? }\end{array}$ \\
\hline 5. Cohesión & $\begin{array}{l}\text { Mecanismo morfosintáctico que permite } \\
\text { ligar una cláusula o preposición, oración } \\
\text { o un párrafo con algún otro elemento } \\
\text { mencionado previamente en otra frase, } \\
\text { cláusula o párrafo. }\end{array}$ & $\begin{array}{l}\text { 1. ¿Mi texto tiene concordancia entre: singular-plural? } \\
\text { 2. ¿Mi texto tiene concordancia entre: masculino- } \\
\text { femenino? } \\
\text { 3. ¿Mi texto tiene concordancia entre: sujeto-verbo? } \\
\text { 4. ¿En mi texto, usé adecuadamente esos } \\
\text { conectores o conectivos? } \\
\text { 5. ¿En mi texto, usé bien los pronombres cohesivos? } \\
\text { (él, ella, éstos, etc). }\end{array}$ \\
\hline 6. Coherencia & $\begin{array}{c}\text { Propiedad semántica (estudio de los significa- } \\
\text { dos) y pragmática (uso de las palabras en un } \\
\text { contexto) del texto, que se refiere a dos tipos } \\
\text { de relaciones lógicas: la existente entre los } \\
\text { conceptos que aparecen en cada oración, } \\
\text { (microestructura), y las existentes entre cada } \\
\text { oración con las otras de la secuencia de que } \\
\text { forman parte, (Macroestructura). }\end{array}$ & $\begin{array}{l}\text { 1. En mi texto, ¿hay concordancia, o } \\
\text { correspondencia, entre el título y el contenido? } \\
\text { 2. ¿Las oraciones no se encadenan entre sí? } \\
\text { (frases aisladas) } \\
\text { 3. ¿En mi texto, se produce un desarrollo a partir del } \\
\text { aporte constante de nueva información? } \\
\text { (No hay repetición de información). } \\
\text { 4. ¿Estoy de acuerdo con todo lo que digo a lo largo } \\
\text { del texto? (No me contradigo) } \\
\text { 5. ¿Los conceptos y contenidos están organizados } \\
\text { en base a principios lógicos y pragmáticos? } \\
\text { 6. ¿Las oraciones expresan relaciones razonables, o } \\
\text { de causa- efecto? } \\
\text { 7. ¿Las oraciones están ligadas por circunstancias o } \\
\text { aspectos que comparten la misma relación o base? } \\
\text { 8. ¿Mi texto está basado en conclusiones generales } \\
\text { que son aceptadas por toda una comunidad o } \\
\text { corresponden con el mundo real (garante)? }\end{array}$ \\
\hline 7. Ortografia & $\begin{array}{l}\text { Se encarga de la normas para la } \\
\text { correcta } \\
\text { escritura. }\end{array}$ & $\begin{array}{l}\text { 1. ¿Qué palabras escribí con letras incorrectas? } \\
\text { (combinación mayúsculas y minúsculas, } \\
\text { mal uso de mayúsculas) } \\
\text { 2. ¿Usé correctamente los signos de } \\
\text { puntuación?(,.:-¿?;!) }\end{array}$ \\
\hline 8. Gráfica & $\begin{array}{c}\text { Se encarga de la disposición de las } \\
\text { palabras en un espacio. }\end{array}$ & $\begin{array}{l}\text { 1. ¿Mi texto presenta errores de segmentación? } \\
\text { (incorrecta separación de palabras) } \\
\text { 2. ¿Mi texto presenta errores de unión incorrecta } \\
\text { de palabras? }\end{array}$ \\
\hline
\end{tabular}




\section{Metodología}

El abordaje del estudio fue de tipo cualitativo, obedeció al método Investigación-Acción, la muestra fue seleccionada de manera casual y fueron los estudiantes de noveno grado del centro educativo "Mimbres" del municipio de Ciénaga de Oro-Córdoba. La hipótesis de acción fue: El desarrollo del programa holístico de metaescritura, produce una mejora en la competencia textual y afectiva de los estudiantes de noveno grado del centro educativo "Mimbres", las categorías de análisis fueron las expuestas en la tabla 2. El estudio se realizó en tres fases: 1. Fase de diagnóstico. 2. Diseño y desarrollo del plan de acción del programa holístico de metaescritura y 3. Evaluación del programa y formulación de nuevas acciones.

Para la puesta en práctica del modelo holístico de metaescritura en el aula de clases, las actividades propuestas en los diferentes talleres del programa (tabla 1), se tuvieron los siguientes pasos (Acción, observaciónreflexión, evaluación y nueva acción), siempre teniendo en cuenta que el programa estuvo centrado en el proceso de revisión - debido a la facilidad que representa para los estudiantes trabajar sobre un texto tangible, y no sobre lo que se encuentra en el pensamiento (MCCORMICK, 1998) el cual a través del resultado del proceso de observación-reflexión y el uso de revisión bibliográfica sobre este proceso - revisión, cambió su nombre por el de revisión de la meta-escritura. A modo de ejemplo podemos citar la siguiente actividad: de forma individual los estudiantes debían escribir un cuento (tabla 1, actividad número 1), donde reinventaran la historia del ave fénix, teniendo como idea central la característica principal del ave (morir y renacer), incluyendo aspectos, circunstancias y/o personajes imaginados o reales. Este cuento debía tener la superestructura correspondiente (inicio, nudo y desenlace). Siguiendo el proceso de la espiral acción, observación-reflexión, evaluación y nueva acción, se presentan los momentos en la actividad antes mencionada de la siguiente manera.

\section{Primer paso - escritura de borradores (Acción):}

En un primer momento, los estudiantes escribieron un borrador de acuerdo al tema y modalidad de texto propuesto en los talleres (tabla 1, actividad número 1). 


\section{Segundo Paso - Autoevaluación (observación-reflexión):}

Para llevar a cabo la autoevaluación, se aplicó el instrumento "formato para revisión de metaescritura" (tabla 2) al borrador que habían escrito. Con la ayuda de lápices de colores que ellos llevaron al salón, debían señalar sus errores, a partir de la siguiente instrucción: los errores de ortografía los pintarían con color azul, los de cohesión con otro color distinto al azul y los de coherencia con uno distinto al de cohesión. Ellos empezaron a leer su cuento, fueron buscando y marcando los errores encontrados en sus escritos (figura 1).

FIGURA 1. Escrito borrador del cuento de un(a) estudiante

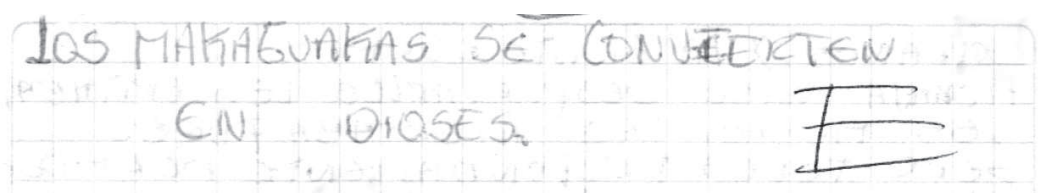

Los maliaguatias salieron un dia hacia el Mon-

te para mitayar lejos del pueblo condlo

UIA UN hURAJA, QUIEN SE ENCENTEO EN El Fonte UNA GRAN COCHA EN UN CANANGU. CHAl.

En aquella cocha Habra eiv abundancra be ZINbo, pERo vro QuE EIU LA MITAD SE EUCONTRABA. UIUA NUNA DE PIES SOBRE WWA FUENTE DE AGUA QUIEN AI VERO LE D. J0:

USTEN UNENE AQUI POR COMIDA PERO NO haY TIAS GUE PESES, PERO COMASE ONO, al INSTANTE QUE FURAJA LO Comio se MURIO EN UN BSTANTE. tos matiagoakas llegaron alä cocha xaluer la gran cantioab ol zingo. DE CHDIERON barbosQUEAR, LOS PESES SALIAN EN TANA ABUNDANCIA QLUE Lle. NARON TODOS SUS CAIVASAOS. CUANDO KLAgARON a SUS CASAS lOS PREPARAROA COMIEROW HOMBKES, MUJERESY NIIVOS. el Hombre llamado huraja se desaPAReCro y al tercer dia resucito CON ROPAS DIFERENTES, Y MUY CSTRANT CON TRASUNTO DE DIOS ANGELICALY DICIENDO QUE HABIA HABIADO CON UnA DIOSA Liamada la diabla.

A pocos istantes les llego a todos ve FUERTE SUENO, TODOS ESTABAN PERJEC-
fatENTE DORHIDOS Y AQVLIA COCHA enpezo a rubir y se oran ruidos 
Cabe anotar que en esa sesión hubo muchas preguntas relacionadas con el formato de revisión puesto que era la primera vez que se enfrentaban a éste. En este caso, se observó la frecuencia de las preguntas respecto a los puntos del formato y se reflexionó sobre este hecho, dando como resultado, el cambio (reformulación de las preguntas) de algunos puntos que los estudiantes no entendieron. Básicamente, en este fase se presentan dos momentos, inicialmente al contrastar lo escrito con los criterios del formato, se busca la autopercepción de errores, es decir un nivel de conciencia que implica un reconocimiento de su error, pero también la identificación del tipo de error en el cual incurrió, esto con miras a realizar la autocorrección de sus errores (autorregulación, CHÁVEZ, 2006, p. 98). Estas dos acciones conforman la autoevaluación. La inclusión de este formato cambia radicalmente el rol del estudiante, ya que le concede un papel activo y protagónico (ATORRESI, 2005).

La fase de revisión se convierte en un momento crucial en la enseñanza de la escritura, esto lo afirma Atorresi (2005, p. 7) en el siguiente texto: "Actualmente, se sabe que la revisión y la autocorrección son procesos fundamentales en la composición del texto. En efecto, los alumnos, si son orientados de manera conveniente, resultan capaces de autocorregirse de la misma manera que de generar ideas". Y justamente ésta es la habilidad que se quiere promover en los estudiantes, a través de la utilización del formato.

\section{Tercer Paso - Evaluación externa}

Co-evaluación. En esta fase, la evaluación se da por parte de otros estudiantes (pares), utilizando el mismo formato, ya que la colaboración entre pares está ampliamente soportada por varios autores (VIGOTSKY, 1995; GRAVES, 1991; MCCORMICK, 1998; OCHOA; ARAGÓN; CORREA; MOSQUERA, 2008) quienes otorgan un papel importante a la interacción social y el soporte de otros compañeros en el aula de clases. Para el caso del ejemplo, no fue aplicada esta fase.

Evaluación por parte del docente: En esta etapa, los investigadores, evaluaron los escritos de los estudiantes utilizando los criterios del formato, los pasaron a la plantilla de Excel $2007^{\circledR}$, y ésta arrojó unos niveles de desempeño sobre el escrito borrador del cuento. Así también, se evaluaron los conceptos relacionados a la autopercepción de los errores de los estudiantes, es decir, se tomaron los datos escritos en el formato llenado 
por ellos (figura 2) y también se sistematizaron en la plantilla, arrojando los niveles de desempeño efectivo autopercibidos propuestos por el decreto 1290 de 2009 - Bajo, Básico, Alto y Superior - (MINISTERIO DE EDUCACIÓN NACIONAL, 2009).

FIGURA 2. Formato de revisión de metaescritura diligenciado por un(a) estudiante

\begin{tabular}{|c|c|c|c|}
\hline Criterios & Definicion & Preguntas Metacoonitivas & Respurstas \\
\hline $\begin{array}{l}\text { Propósito } \\
\text { Comunicativo }\end{array}$ & $\begin{array}{l}\text { Intención comunicativa } \\
\text { especifica por la que se } \\
\text { escribe el texto. }\end{array}$ & $\begin{array}{l}\text { 1. Para qué escribi el texto? } \\
\text { 2. ¿Se vé claramente en el texio el } \\
\text { propósitio por el cuaral lo escribi? }\end{array}$ & 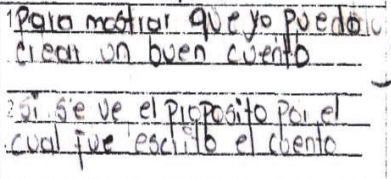 \\
\hline Sentido Completo & $\begin{array}{l}\text { Cuando en el texto } \\
\text { aparece } \\
\text { información para que al } \\
\text { leerlo se pueda entender } \\
\text { el proposilio por el cual se } \\
\text { escribio. }\end{array}$ & $\begin{array}{l}\text { 1. CTiene el lexto suficiente } \\
\text { intomación para que el lector } \\
\text { comprenda mi proososito? }\end{array}$ & 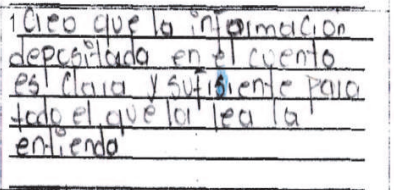 \\
\hline \multirow{2}{*}{ Unidad temática } & \multirow{2}{*}{$\begin{array}{l}\text { Cuando en el texio e ltema } \\
\text { se desarrolla con una idea } \\
\text { principal y o ditas } \\
\text { secundarias, además, no } \\
\text { se incluye información que } \\
\text { no esté directamente } \\
\text { relacionada con el tema. }\end{array}$} & \multirow{2}{*}{ 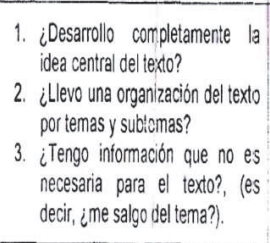 } & $\begin{array}{l}15 \% \text { desaliollo laidea mairy } \\
\text { yienacer } \\
\text { no aplia }\end{array}$ \\
\hline & & & $\begin{array}{l}\text { 3no ten } 90 \text { in famacion que no } \\
\text { tenga que uel con eltexto }\end{array}$ \\
\hline \multirow{6}{*}{ Coherencia } & \multirow{6}{*}{ 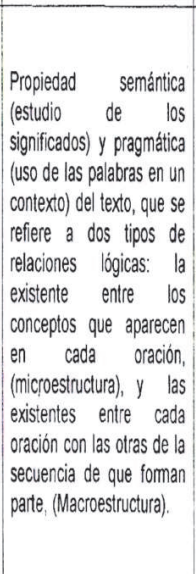 } & \multirow{6}{*}{ 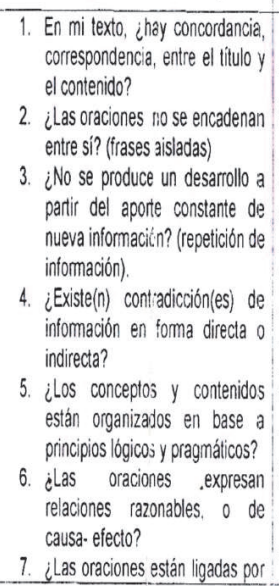 } & $\begin{array}{l}\text { no condoenda ellitulo CoD } \\
\text { el texto } \\
\text { 2clare que estan bien unidas }\end{array}$ \\
\hline & & & $\begin{array}{l}\text { 3congidelo que la infamacion } \\
\text { otiene lepeticion }\end{array}$ \\
\hline & & & $\begin{array}{l}\text { 4no exigte ninguna contani. } \\
\text { cion }\end{array}$ \\
\hline & & & smi cuento si tiene lol \\
\hline & & & 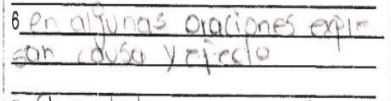 \\
\hline & & & 7. El relaio a e a/1 \\
\hline
\end{tabular}

Los datos fueron copiados y analizados en el paquete estadístico SPSS 15.0 para Windows.

\section{Cuarto Paso - Reescritura (nueva acción).}

Este es el momento final, donde se integra la autoevaluación, la Co-evaluación - si se llevó a cabo - y la evaluación del docente, para dar lugar a un nuevo texto, donde se hayan corregido los errores encontrados (figura 3). 
FIGURA 3. Escrito final del o la estudiante, después de realizada la evaluación
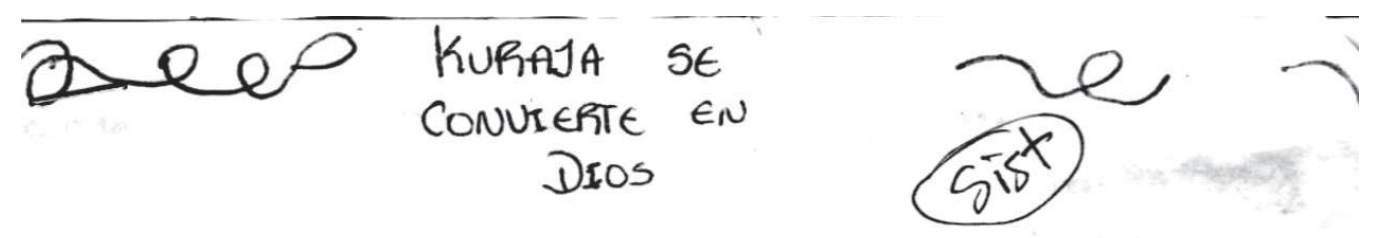

Los makaguatias salieron un dia Hacia el monte para pescar lejos del pueblo, con ellos iba un furaja quien se encontro una gran cocho en w. cananguchal.

En aquella cocha habia abundancia de peces; pero vio que en la hitad se encontrabo una niña de pies, sobrat ono fuente de ajua quien al verlo le dijo:

Usted siene aqui por comida pery to hay mas que peces.si quigie comase ono al insfant que cuando la tribu if ego y vierop la gran fantidad de peces, decidieros llevarsfolos. Salian tantos que llenaron todos sus capostos, ql Iyegar

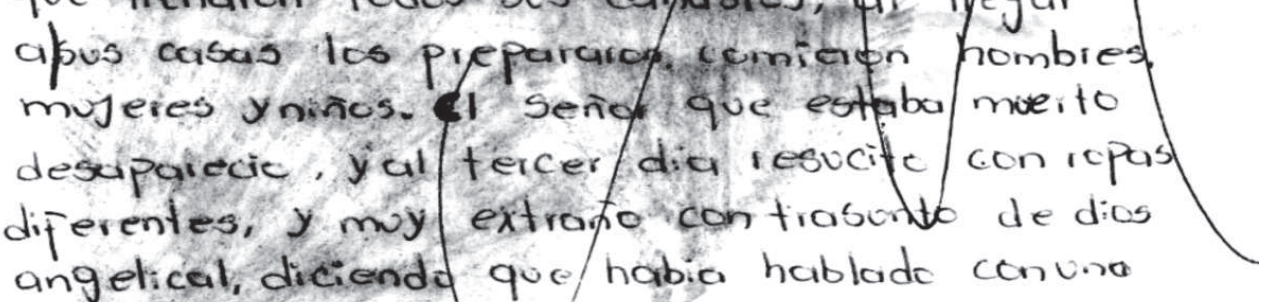
diosa llamada la diabla.

A pocos momeritos tes llega a tados on fuerte sueño, todos estaban profundamente dormidos. y aquel lugar empezo a rugir y se cian ruidos, y aquel hombre quise despertarlos. les gritaba, los movia, les acercaba fuego pero todos perfectamente dormidos, y los dicses del mas alla les sacarón los ojos...

Para el caso del ejemplo, una vez revisados los escritos de los estudiantes por ellos mismos y por el profesor, la docente procede a devolvérselos para que ellos, reescribieran el cuento. Una vez terminado el cuento (escrito final - figura 3), se le realizó también el análisis con el formato de revisión de metaescritura y se sistematizó en la plantilla elabo- 
rada en Excel destinada para tal fin, luego, se procedió al análisis necesario para comprobar qué tanto fue el impacto de la aplicación del formato de revisión de metaescritura.

Para finalizar podemos decir que la escritura se convierte en un medio y en un fin; en medio, cuando es utilizada para lograr propósitos relacionados con otros usos, y en fin cuando se ejerce un control sobre los diferentes elementos teóricos-conceptuales que la conforman, con el propósito de evaluarse y mejorarse a sí misma. Toda la estructura de la metodología del programa holístico puede ser resumida en el gráfico 2:

GRÁFICO 2. Operacionalización de la metodología del Modelo Holístico de metaescritura

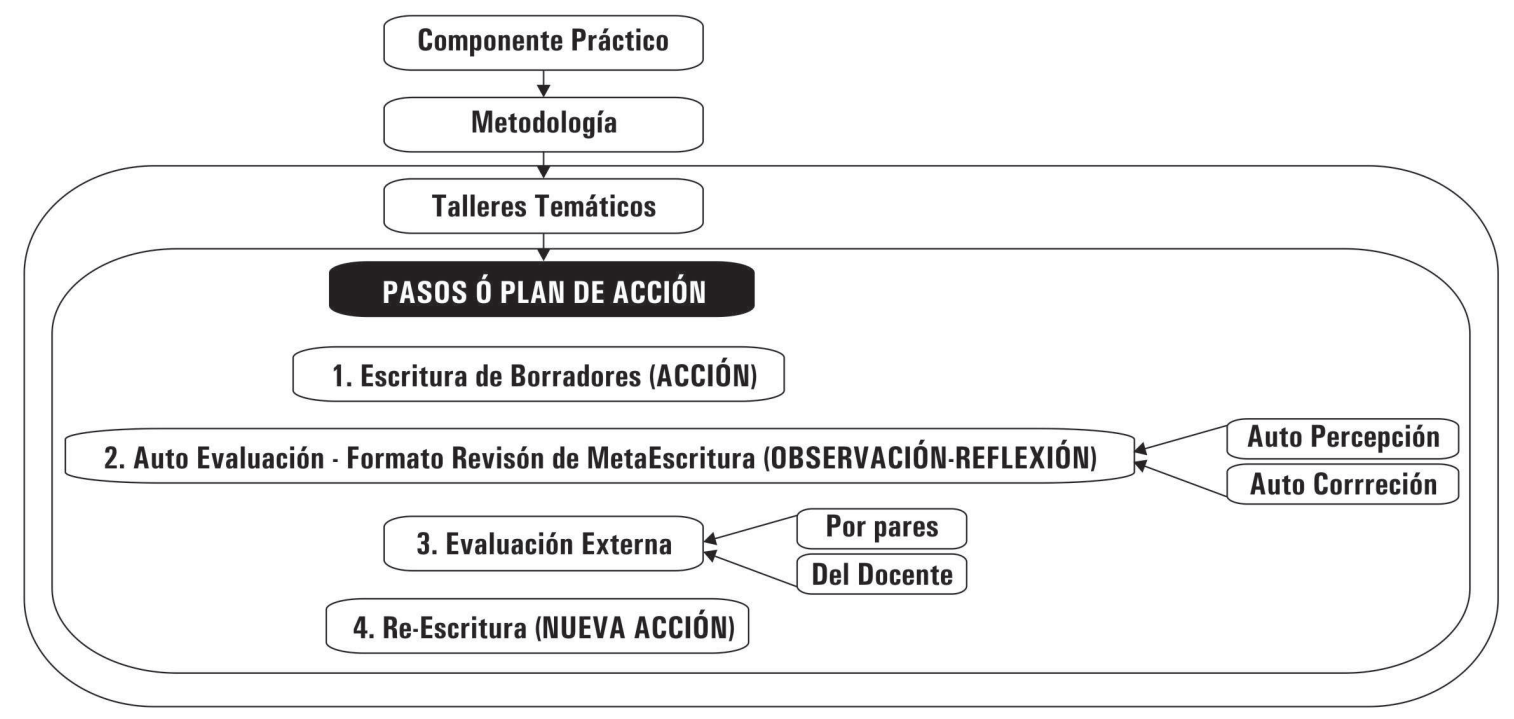

Resultados obtenidos

A nivel teórico

Como consecuencia del estudio de diversas teorías y de la necesidad de especificar un concepto que abarque la articulación de los elementos teóricos realizada por el equipo investigador, se dio origen al concepto de Metaescritura. El recorrido hacia éste, se inició con el término metalenguaje, el cual fue definido como "una actividad lingüística que tiene como objeto el propio lenguaje" (JACOBSON, 1963, citado en MAYOR, 1993, p. 72), pero el término metalenguaje evolucionó hacia uno más amplio Habilidad metalingüística (MAYOR, 1993, p. 73). 
Éste término es de gran utilidad, debido a que fusiona la metacognición y la lingüística. Por lo que respecta a la metalingüística, en cualquier caso incorpora las actividades de reflexión y control sobre todos los componentes lingüísticos. Dando lugar a que se subdivida en metafonología, metasintaxis, metasemántica, y en su caso, metapragmática, (incluso metaléxica y metatextual) (GOMBERT, 1990 citado en MAYOR, 1993, p. 74). De lo anterior, se acoge el termino habilidad metatextual, la cual "implica la capacidad de ser conscientes y para controlar la producción y la comprensión de textos" (MAYOR, 1993, p. 140).

Los estudios realizados respecto a la habilidad metatextual muestran que el control consciente sobre los componentes textuales no surge espontáneamente, sino que necesita una instrucción específica para que pueda llevarse a cabo, tomando en cuenta lo anterior, y las interrelaciones que se han establecido entre las diferentes dimensiones que se han analizado, se encontró que se puede hacer una nueva definición del concepto de escritura, que haga una integración de estas dimensiones, pero proponiendo la metacognición como elemento transversal, por tanto, aplicada a cada una de las dimensiones estudiadas. Es decir, una conciencia sobre el aspecto afectivo-emocional que se está expresando, sobre los procesos de pensamiento y conocimientos empleados, así como la comprensión de los elementos contextuales emergentes y por último el manejo de las categorías textuales a tener en cuenta para estructurar la escritura.

Con el fin de establecer diferencias entre el anterior concepto y esta nueva construcción se formula el término "metaescritura" entendida como la habilidad especifica de ser consciente y controlar los elementos textuales, cognitivos, afectivo-emocionales y contextuales, que se activan y movilizan al momento de escribir. Esto se puede visualizar en el gráfico 1:

A nivel práctico, se organizaron desde las dos competencias trabajadas, la textual y la emocional. Respecto a la competencia textual, se comparó el desempeño obtenido en la realización del borrador (figura 1) y el escrito final (figura 3), después de utilizar el formato para revisión de metaescritura (figura 2). Los datos obtenidos de la plantilla de evaluación de niveles de desempeño en las frecuencias para las diferentes categorías de esta competencia, fueron procesadas estadísticamente en el software SPSS, versión 15.0 para Windows. 
Resultados niveles de desempeño en la competencia textual

Los resultados del ejemplo elaborado en el taller 1 (tabla 1, actividad 1) obtenidos por el software SPSS $15.0^{\circledR}$ para las distintas dimensiones propuestas en las categorías de análisis fueron:

TABLA 3. Resultados de los niveles de desempeño en la competencia textual

\begin{tabular}{|c|c|c|c|c|c|c|}
\hline NIVEL & FRECUENCIA & $\begin{array}{l}\text { PORCENTAJE } \\
\text { VÁLIDO }\end{array}$ & $\begin{array}{l}\text { PORCENTAJE } \\
\text { ACUMULADO }\end{array}$ & FRECUENCIA & $\begin{array}{l}\text { PORCENTAJE } \\
\text { VÁLIDO }\end{array}$ & $\begin{array}{l}\text { PORCENTAJE } \\
\text { VÁLIDO }\end{array}$ \\
\hline \multicolumn{7}{|c|}{ NIVEL DE DESEMPEÑO SUPERESTRUCTURA } \\
\hline Bajo & 1 & 6,3 & 6,3 & . & . & . \\
\hline Superior & 15 & 93,8 & 100,0 & 16 & 100,0 & 100,0 \\
\hline Total & 16 & 100,0 & . & 16 & 100,0 & . \\
\hline \multicolumn{7}{|c|}{ NIVEL DE DESEMPEÑO PROPÓSITO COMUNICATIVO } \\
\hline Bajo & 3 & 18,8 & 18,8 & . & - & - \\
\hline Básico & 13 & 81,3 & 100,0 & 16 & 100,0 & 100,0 \\
\hline Total & 16 & 100,0 & & 16 & 100,0 & . \\
\hline \multicolumn{7}{|c|}{ NIVEL DE DESEMPEÑO SENTIDO COMPLETO } \\
\hline Bajo & 3 & 18,8 & 18,8 & . & - & . \\
\hline Básico & 3 & 18,8 & 37,5 & . & . & . \\
\hline Superior & 10 & 62,5 & 100,0 & 16 & 100,0 & 100,0 \\
\hline Total & 16 & 100,0 & . & 16 & 100,0 & . \\
\hline \multicolumn{7}{|c|}{ NIVEL DE DESEMPEÑO UNIDAD TEMÁTICA } \\
\hline Bajo & 1 & 6,3 & 6,3 & 3 & 18,8 & 18,8 \\
\hline Básico & 15 & 93,8 & 100,0 & . & . & - \\
\hline Superior & . & . & . & 13 & 81,3 & 100,0 \\
\hline Total & 16 & 100,0 & & 16 & 100,0 & \\
\hline \multicolumn{7}{|c|}{ NIVEL DE DESEMPEÑO COHESIÓN } \\
\hline Bajo & 5 & 31,3 & 31,3 & 2 & 12,5 & 12,5 \\
\hline Básico & 7 & 43,8 & 75,0 & 2 & 12,5 & 25,10 \\
\hline Alto & 3 & 18,8 & 93,8 & 7 & 43,8 & 68,8 \\
\hline Superior & 1 & 6,3 & 100,0 & 5 & 31,3 & 100,0 \\
\hline Total & 16 & 100,0 & . & 16 & 100,0 & . \\
\hline
\end{tabular}

Análisis explicativo

Como se puede observar en la tabla anterior, es posible decir que sí hubo transformaciones positivas debido a la utilización del formato de revisión de metaescritura como herramienta didáctica, el cual está orientado a que los estudiantes autoperciban y autocorrijan los errores presentados por ellos en la producción de sus textos.

Respecto al análisis de la competencia emocional, se puede observar:

Una de las inspiraciones para el trabajo de investigación surgió 
de la lectura de diversos textos que proponían talleres de escritura donde los estudiantes incluían como material de trabajo, su vida, sus problemas, temores, alegrías, intereses, sueños, sentimientos y pensamientos, entonces se pensó: ¿Por qué no hacer realidad esto en nuestras aulas y aplicar en la escuela los usos que se muestran en algunas investigaciones, para comprobar si realmente podía tener tanta importancia para nuestros alumnos de carne y hueso, rodeados de un contexto especifico, - en este caso rural y de pobreza - y si tenía los mismos efectos que proponían algunas aproximaciones a la escritura?.

Inicialmente se tratará la escritura con la funcionalidad de expresión de sentimientos e instrumento catártico. En este aspecto, se pudo observar que los estudiantes sí emplearon la escritura para sacar fuera de sí mismos, situaciones traumáticas y dolorosas que le habían ocurrido, lo cual puso de manifiesto la utilidad de la escritura para hacer conscientes y elaborar situaciones difíciles o desagradables.

A continuación, se muestran algunas de las opiniones expresadas por los estudiantes, respecto a los beneficios de la escritura:

"En ella he podido desahogarme y hablar de algunas cosas que han sido importantes en mi vida".

"He podido expresar mi sentimiento de tristeza, de alegría que no habia expresado por miedo".

"forma de revelar aquellos problemas que no nos dejan vivir tranquilo, podemos expresar sentimientos y dirigirnos a una persona, y despejar nuestra mente y mantenernos más relajados.

Los estudiantes han escrito acerca de varios temas que están directamente relacionados con sus vidas, esto está basado en las múltiples propuestas desde diferentes autores de utilizar lo que realmente es importante para ellos, que está conectado a sus vivencias, a su contexto y su realidad interna, y en general a las vicisitudes acontecidas en las historias de sus vidas.

Entre las situaciones acerca de las cuales ellos han escrito, se encuentran:

Las vivencias y dificultades acaecidas en la escuela, y la percepción que tienen de los docentes.

"A veces me iba para el colegio, sin comer, sin merienda, mi bolso era una mochilita de tela que me fabricaba mi mamita los profesores eran muy duros, si no nos sabiamos las tablas nos castigaban (reglasos - jalones de oreja - nos arrodillaban sobre unas checas [tapa de botella de gaseosa] y en el sol." 
El dolor por la separación de los padres,

"Cuando yo tenia 9 años de edad mis padres se separaron por primera vez, pensé que hay acababa mi vida me quería morir pues me sentía sola y mis hermanos también sufrían."

El maltrato físico y verbal por parte de los padres:

"Todo el tiempo de mi vida que pase en mi casa era puro regaños, malos maltratos y asta me golpeaba mi mama, ella todo el tiempo se la pasaba gritándome... mucha gente le inventaba tantas barbaridades que asta llegaba a golpearme muy duro, y eso me dolia y mucho me decía entre mi porque la persona que yo mas quería me trataba tan mal y dije esto no lo puedo soportar mas tengo que salir de este infierno."

Así mismo, han escrito acerca de las abuelas, del padre, pérdida de un bebé, de desaparición de familiares, peleas con las amigas, peleas entre familiares, entre otros aspectos. Con todo lo anterior como soporte, se puede erigir la escritura como un elemento catártico que proporciona un beneficio a nivel psicológico ya que coadyuva la superación de problemáticas personales, que en algunos casos no tienen ninguna otra forma de trabajarse, yaciendo aparentemente inertes en el interior del individuo, pero que en cualquier momento pueden activarse y traer obstáculos al sano desarrollo de la personalidad de los estudiantes.

Con referencia al componente metacognitivo, los siguientes apartes expresan las opiniones de los estudiantes respecto al uso de la metacognición:

"Me ayudo mucho, puedo corregir los errores que encuentro en mi camino", "Ayuda a encontrary corregir errores", "Puedo saber yo misma que tanto pienso", "sabiendo mi autoconocimiento que he aprendido en los talleres de metacognitivo y los errores que tengo en el formato", "Cometo pequeños errores pero gracias a lo que aprendí tengo la facilidad de corregirlos".

Asi mismo los estudiantes reportan una conciencia de su estado y avances en el proceso escritural. Al respecto, los estudiantes manifestaron que mejoraron en los siguientes elementos:

"reglas de ortografia", "clases de narraciones", "Usar las palabras y los conectores correctamente", "Las cosas siempre tienen un propósito para el lector, y es que comprenda", "Crear cuentos, relatos, noticias, canción", "Mejorar errores en mi escritura". 
Es relevante la continua alusión que hacen los estudiantes a la ortografía, lo cual puede ser efecto del continuo énfasis que se ha hecho en los años anteriores, desconociendo otros factores, que solo fueron introducidos durante este año, sin embargo, en algunos se nota la referencia a otros aspectos tales como:

"Ahora he aprendido escribir y elaborar un texto o cualquier tipo de escrito y no tengo tantos errores a la hora de escribir", "ayuda a corregir errores ortográficos, información contradictoria, si tenemos contenidos de mas, si utilicemos bien los conectivos".

Esto se considera un avance, porque los estudiantes empezaron a ser conscientes de la utilización de otros aspectos en su escritura, lo cual ayuda a que tengan una visión más amplia de su desempeño como escritores.

\section{Conclusiones}

La metodología aplicada en esta investigación, es decir, la propuesta de talleres, el método de enseñanza (escritura del borrador, revisión por el estudiante, el profesor y/o los pares, reescritura del texto final) y la herramienta didáctica "formato de revisión de metaescritura", sí ayuda a los estudiantes a mejorar sus niveles de desempeño efectivo en su competencia textual.

Utilizar marcadores gráficos para resaltar los errores encontrados con códigos de colores en el escrito borrador (monitoreo), facilita a los estudiantes hacer las respectivas correcciones (control) en sus textos finales.

La escritura sí puede emplearse exitosamente en los estudiantes para expresar y elaborar situaciones traumáticas, dolorosas o estresantes, por tanto se confirma su función catártica y su utilidad para fortalecer la competencia afectivo-emocional de los estudiantes (PENNEBAKER; CHUNG, 2007).

Una enseñanza eficaz y productiva de la escritura debe incluir ineludiblemente el componente de la metacognición, ya que la conciencia y el control de los procesos escriturales son indispensables si se quiere transformar las prácticas de escritura vivenciadas en nuestras escuelas (PÉREZ, 1998). 
Con el modelo holístico de escritura y la investigación-acción se puede lograr y ayudar a entender el todo, a una comprensión integral del ser humano, no sólo como sujeto que aprende sino que siente, que piensa, que se expresa y vive una realidad concreta, no se trata de ser sólo investigador, se trata de estar inmerso en la realidad del contexto donde habitan nuestros estudiantes, es sentir, vibrar, gritar, jugar y sobre todo entender a nuestros estudiantes.

Con la metodología implementada en la investigación, los estudiantes pudieron emancipar sus sentimientos reprimidos, expresar sus pensamientos, crear nuevas ideas, nuevos mundos, sentir que estaban vivos y que alguien los escuchaba a través de la voz de las letras.

El proceso de escritura es complejo, dado que involucra la dimensión afectivo-emocional, una dimensión cognitiva, competencia textual y utiliza procesos metacognitivos enmarcados dentro de un contexto sociocultural específico.

\section{Recomendaciones}

Se debe enseñar y aplicar en las escuelas la Metacognición como un eje transversal que abarque todas las áreas del saber y en especial el área de Lengua Castellana, con énfasis en la lectura y en la escritura.

Se recomienda el uso por parte de los docentes en ejercicio desarrollar el programa holístico de metaescritura para fomentar en sus estudiantes el nivel de control y conciencia del proceso escritor, lo cual contribuirá a mejorar dichos procesos. 


\section{Referencias}

ASOCIACIÓN COLOMBIANA DE FACULTADES DE EDUCACIÓN [ASCOFADE]. Estándares básicos de Competencias en Lenguaje, matemáticas, Ciencias y Ciudadanías. Santafé de Bogotá: Ministerio de Educación Nacional, 2006.

ATORRESI, Ana. Construcción y Evaluación de consignas para evaluar la escritura como competencia para la vida. Revista Enunciación, 10, p. 4-14, 2005.

BARA, Pedro Mariano. Estrategias metacognitivas y de aprendizaje: estudio empírico sobre el efecto de la aplicación de un programa metacognitivo; el dominio de las estrategias de aprendizaje en estudiantes de ESO, BUP y universidad. Tesis (Doctorado para optar al título de Doctor, Departamento de didáctica y organización escolar). Facultad de educación, Universidad Complutense de Madrid, Madrid, España, 2001.

BRUDER, Mónica. El cuento terapéutico como favorecedor de la resiliencia. Una primera aproximación. Psicodebate 6. Psicología y salud, p. 15-28, 2007. Disponible en: <http://www.palermo.edu/cienciassociales/publicaciones/pdf/Psico6/6Psico\%2002.pdf>. BRUNNER, Jerome. Realidad mental y mundos posibles: los actos de la imaginación que dan sentido a la experiencia. Barcelona: Editorial Gedisa S.A, 2004.

CHÁVEZ, José M. Guía para el desarrollo de los procesos metacognitivos. Lima: Kinko's impresores S.A.C. Ministerio de Educación República del Perú, 2006. Disponible en: $<$ http://destp.minedu.gob.pe/secundaria/nwdes/pdfs/Guiaprocesosmetacognitivos.pdf $>$. CASTILLO, Martha Janeth; TRIANA, Norma; DUARTE, Nohora; PÉREZ, Mauricio; LEMUS, Eduardo. SABER: Subdirección académica Grupo evaluación de la educación Básica y Media. Área: Lenguaje. Análisis de Preguntas Aplicación 2005-2006. Santafé de Bogotá. D.C. Ministerio de Educación Nacional [M.E.N.], 2007. Disponible en: <http://menweb.mineducacion.gov.co:8080/saber/analisis_preguntas_Lenguaje_2005-2006.pdf>.

DÍAZ, Álvaro. Aproximación al texto escrito. 4 ed. Medellín: Caminos, Universidad de Antioquia, 1999.

ESCUELA DE ESCRITORES. Escribes. Escritura terapéutica, 2009. Disponible en: <http://escribes.es/5/Escritura-terapeutica.html>. Extraído en: 8 Oct. 2009.

FERREIRO, Emilia; GÓMEZ, P. M. (Comp.) Nuevas perspectivas sobre los procesos de lectura y escritura. Buenos Aires: Siglo XXI Editores, 2002.

FERREIRO, Emilia. Cultura escrita y educación. Conversaciones con Emilia Ferreiro. México: Fondo de Cultura, 1999.

FLOWER, Linda; HAYES, John. La teoría de la redacción como proceso cognitivo. En: G. MESTRONI (Trad.), M.E. RODRÍGUEZ (Ed.) La teoría transaccional de la lectura y la escritura. Buenos Aires: Asociación Internacional de Lectura, 1996.

GRAVES, Donald H. Didáctica de la escritura. 12 ed. Madrid: Ediciones Morata S.A., 1991. GROTBERG, Edith. Resilience for today. Gaining strenguth from adversity. (Comp.) En: GARCÍA, A. (Trad.). La resiliencia en el mundo de hoy: cómo superar las adversidades. Barcelona: Gedisa, 2006.

HERNÁNDEZ, Yamid Fabián; SERPAS, Yasmín Osmany. Programa Holístico de Escritura para fortalecer la competencia textual y emocional en estudiantes de noveno grado del centro educativo "Mimbres" de Ciénaga de Oro. Trabajo de grado para optar al título de Magíster en Educación, Facultad de Educación y Ciencias Humanas, Universidad de Córdoba, Montería, Colombia, 2009. 
LACON, Nelsi; ORTEGA, Susana. La problemática de la escritura en la universidad: una propuesta de solución a partir de la articulación con el Polimodal. I CONGRESO INTERNACIONAL EDUCACIÓN, LENGUAJE Y SOCIEDAD: “TENSIONES EDUCATIVAS EN AMÉRICA LATINA”. Santa Rosa, La Pampa, Argentina: Facultad de Ciencias Humanas, Universidad Nacional de La Pampa. Libro digital, 2004. Disponible en: $<$ http://www.unne.edu.ar/institucional/documentos/lecturayescritura08/lacon_ortega.pdf >. MARINKOVICH, Juana. Enfoques de proceso en la producción de textos escritos. Rev. Signos, v. 35 n. 51-52 Valparaíso, 2002. Disponible en:

<http://www.scielo.cl/scielo.php?pid=S0718-09342002005100014\&scrip >. Extraído en: 10 Sept. 2009.

MAYOR, Juan. Estrategias metacognitivas: aprender a aprender y aprender a pensar. Madrid: Editorial Síntesis, 1993.

McCORMICK, Lucy. Didáctica de la escritura en la escuela primaria y secundaria. Buenos Aires: Aique, 1998.

MINISTERIO DE EDUCACIÓN NACIONAL. Decreto n. 1290, 2009. Bogotá, D.C. Disponible en:

<http://www.mineducacion.gov.co/1621/articles-187765_archivo_pdf_decreto_1290.pdf >. Extraido en: jun. 2009.

MORALES, Felipe; FONSECA, Clara Inés; MORALES, Julio; MORALES, Enrique. Leer y escribir en la universidad. Bogotá D.C.: Guadalupe, 2006.

OCHOA, Solanlly; ARAGÓN, Lucero; CORREA, Miralba; MOSQUERA, Santiago. Funcionamiento metacognitivo de niños escolares en la escritura de un texto narrativo antes y después de una pauta de corrección conjunta. Acta Colombiana de Psicología, v. 11, n. 2, p. 77-88, 2008. Disponible en:

<http://portalweb.ucatolica.edu.co/easyWeb2/files/23_1278_v11n2-art7.pdf>.

PENNEBAKER, J.W.; CHUNG, C.K. Expressive Writing, Emotional Upheavals, and Health. In: FRIEDMAN, H.; SILVER, R (Eds.) Handbook of health psychology. New York: Oxford University Press, 2007. p. 263-284. Disponible en:

<http://homepage.psy.utexas.edu/HomePage/Faculty/Pennebaker/Reprints/Penneba ker\&Chung_FriedmanChapter.pdf $>$.

PEÑA, Luis Bernardo. La escritura como una forma de reivindicar el saber de los maestros. En: PENAGOS, Rafael Ávila (Comp). La investigación - acción pedagógica: experiencias y lecciones. Bogotá D.C.: Antropos, 2003. Disponible en:

<http://www.cerlalc.org/Escuela/datos/escritura.doc>. Extraído en 7 sept. 2009.

PÉREZ, Mauricio. Leer y escribir en la Escuela: Algunos escenarios didácticos y pedagógicos para la reflexión. Instituto Colombiano para el Fomento de la Educación Superior [ICFES], 2003. Disponible en:

<http://www.cerlalc.org/Escuela/enlaces/M_Perez_Leer_y_escribir_escuela.pdf $>$. Extraído en 12 sept. 2009.

PÉREZ, Mauricio. Lengua castellana: lineamientos curriculares. Santafé de Bogotá, D.C.: Cooperativa Editorial Magisterio. Ministerio de Educación Nacional, 1998.

ROCKWELL, E. Los usos escolares de la lengua escrita. En FERREIRO, Emilia; GÓMEZ, P. M. (Comp.). Nuevas perspectivas sobre los procesos de lectura y escritura. Buenos Aires: Siglo XXI, 2002.

RODRÍGUEZ, Ada, Escritura, Cognición y Metacognición. QUINTO CONGRESO 
INTERNACIONAL DE LA CÁTEDRA UNESCO PARA EL MEJORAMIENTO DE LA CALIDAD Y EQUIDAD DE LA EDUCACIÓN EN AMÉRICA LATINA, CON BASE EN LA LECTURA Y LA ESCRITURA: El dominio discursivo como ejercicio de la democracia. Instituto Pedagógico de Caracas, Venezuela, 17-19 de Junio 2009. [Abstract] Disponible en: <http://www.catedraunescovenezuela.org/vcongreso/programa/programadef/program_def_archivos/Resumenes $\% 20$ Ponencias $\% 20$ Orales/Ro dr\%C3\%ADguez\%20Ada.doc>. Extraído en 6 Julio 2009.

ROSENBLATT, L. The transactional theory of reading and writing and a cognitive process theory of writing. En: MESTRONI, G. (Trad.), RODRÍGUEZ, M. E. (Ed.). La teoría transaccional de la lectura y la escritura. Buenos Aires: Asociación Internacional de lectura, 1996.

UNITED NATIONS EDUCATIONAL, SCIENTIFIC AND CULTURAL ORGANIZATION [UNESCO]. Declaración de la Habana-Cuba, 2002 . Disponible en: <http://www.systac.cl/archivospsp/habana.pdf>. Extraído en 7 Sept. 2009.

VAN DIJK, Teun. Estructuras y funciones del discurso: una introducción interdisciplinaria a la lingǘstica del texto y a los estudios del discurso (2 ed.) Trad. Myra Gann. México; Bogotá: Siglo Veintiuno Editores, 1983.

VIGOTSKY, Lev S. Pensamiento y lenguaje. Buenos Aires: Ediciones Fausto, 1995.

VILLARINI, Ángel. Manual para la enseñanza de destrezas de pensamiento. San Juan: Pell, 1991.

Recebido: $22 / 03 / 2010$

Aprovado: 22/05/2012

Contato:

Universidad de Córdoba

Facultad de Educación

Dpto de Psicopedagogía

Carrera 6

No $76-103$

Córdoba

Colombia 